\title{
Y-chromosomal haplogroups in male identification: study of a population sample from Portugal (central area)
}

\author{
L. Andrade ${ }^{\text {a }}$, F. Balsa ${ }^{\text {a }}$, V. Lopes ${ }^{\text {a }}$, M.J. Anjos ${ }^{\text {a }}$, M. Carvalho ${ }^{\text {a }}$, \\ F. Corte-Real ${ }^{\mathrm{a}, \mathrm{b}}$, D.N. Vieira ${ }^{\mathrm{a}, \mathrm{b}}$, M.C. Vide ${ }^{\mathrm{a}, *}$ \\ anstituto Nacional de Medicina Legal, Delegação de Coimbra, Serviço de Biologia e Genética Forense, \\ Largo da Sé Nova, 3000-213 Coimbra, Portugal \\ ${ }^{\mathrm{b}}$ Faculty of Medicine, University of Coimbra, Coimbra, Portugal
}

\begin{abstract}
Background: The non-recombining portion of the $\mathrm{Y}$ chromosome represents a valuable tool for the study of human population history. The haploid, non-crossing-over behaviour of Y chromosome gives potentials in many aspects resembling the maternally inherited mtDNA. The advantages for forensic casework are obvious. The haploid state makes mixture patterns much simpler; in female/ male stain mixtures, pure male DNA profiles may be obtained. Y-chromosome markers are also of extraordinary value to solve selected paternity cases. Methods: Y-chromosomal biallelic markers have been investigated in a population sample from central Portugal $(n=117)$. By combining the allelic state of 10 biallelic markers (YAP-DYS287, SRY-8299, 92R7, 12f2, SRY-1532, SRY-2627, Tat, SY81, M9, LLY22g), we could define the haplogroup to which each sample belonged. Results: This population defined 10 different haplogroups, haplogroup 1 being the most frequent. Conclusion: This study showed a substantial similarity with other Iberian studies; however, this population presents a larger diversity.
\end{abstract}

(C) 2003 Elsevier Science B.V. All rights reserved.

Keywords: Y chromosome; Biallelic markers; Haplogroups

\section{Introduction}

Biallelic markers usually arise from unique mutational events (base substitutions, insertions/deletions) and are binary in nature. The interpretation of haplogroups may be

\footnotetext{
* Corresponding author. Tel.: +351-239-854-230; fax: +351-239-820-549.

E-mail address: mcvide@ci.uc.pt (M.C. Vide).
} 
useful for illuminating the substructure of a population or the Y-chromosomal origin of an individual, through the definition of paternal lineages which can be related to one another by a single mostly parsimonious tree. These markers have been widely used for human evolutionary studies and population genetics.

There is increasing interest in the use of biallelic markers for forensic purposes: deficiency paternity testing and discrimination of stains in investigations with male suspect cases. However, it is important to notice that the analysis of biallelic loci for human identification requires the simultaneous analysis of at least 50 loci, regarding the low discriminating power of each locus [1].

\section{Material and methods}

DNA was extracted from blood stains collected from 117 unrelated Portuguese (central area) males, by Chelex 100 method [2].

Ten biallelic markers (eight single nucleotide polymorphisms, SNPs, the Alu insertion YAP-DYS287 and the $12 \mathrm{f} 2$ deletion) were studied in a population from central Portugal.

Biallelic markers were amplified by PCR and SNPs were also analysed by RFLP through an enzymatic digestion with the respective restriction enzyme. SRY1532, DraIII [3]; SRY8299, BsrBI [3]; M9, HinfI [4]; YAP-DYS287 [5]; 92R7, HindIII [6]; SRY2627, BsiHKAI [7]; TAT, NlaIII [8]; SY81, NlaIII [9]; LLY22g, HindIII [10]; $12 f 2$ [11].

Fragments were detected by electrophoresis in PhastGel ${ }^{\mathrm{TM}}$ Gradient 10-15, with PhastGel $^{\mathrm{TM}}$ SDS buffer strips (PhastSystem ${ }^{\mathrm{TM}}$-Amersham Pharmacia Biotech). Fragment length was determined through comparative analysis with a 25- or 123-bp DNA ladder (Gibco BRL $\left.{ }^{\circledR}\right)$.

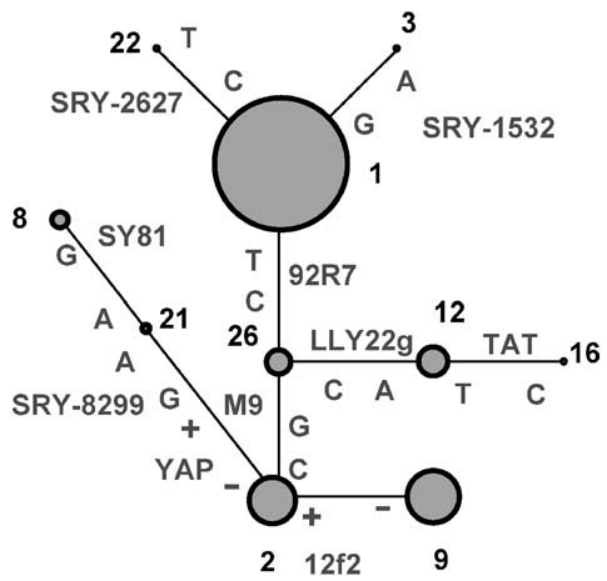

Fig. 1. Distribution of the haplogroups in the Portuguese population (central area); the biallelic markers analysed are indicated along the lines together with the two bases found, the absence/presence of the retroposon insertion YAP or the insertion/deletion at the $12 \mathrm{f} 2$ locus; the relative frequencies are proportional to the areas of the circles. 


\section{Results}

The 10 biallelic markers used in our study together allow the definition of 12 haplogroups. The population of central Portugal defined 10 different haplogroups (Fig. 1).

\section{Discussion}

Haplogroups are found at different frequencies in the European populations; haplogroups that are rare or absent from Europe can be common elsewhere, thus providing markers for identifying migration from these regions [12].

In Iberia, different populations with different languages and history coexist. Comparisons with other Iberian samples analysed for the same markers showed a substantial similarity [13]; however, our study presents a larger diversity.

The population of central Portugal defined 10 different haplogroups, haplogroup 1 being the most frequent.

Haplogroup 22 has a typical presence in the Iberian Peninsula [14].

Haplogroups 12 and 16, not commonly present in Iberian samples, show a wide geographical distribution and are found in Southern and Eastern Asia, but they have not been detected in Africa [8].

Haplogroup 3 is probably very old; it gave rise to most $\mathrm{Y}$ chromosomes found outside Africa [15].

The Y chromosome is thus a sensitive marker for population substructuring and may be useful for determining whether two population samples come from a single population.

Population surveys based in Y-chromosome haplotypes and haplogroups (STRs and biallelic marker loci) seem to be the best strategy for the use of $\mathrm{Y}$ polymorphisms in population genetics, evolutionary studies and in forensic applications [16].

The study of $\mathrm{Y}$ haplotypes is being done for a more detailed information about this population; also studies on other Iberian and European populations are necessary for a detailed interpretation of the data.

\section{Acknowledgements}

The authors would like to thank the technical support of Professor Angel Carracedo (Santiago de Compostela, Spain) and Maria Brión.

\section{References}

[1] P. Gill, Int. J. Leg. Med. 114 (2001) 204-210.

[2] P.S. Walsh, et al., BioTechniques 1 (1991) 91-98.

[3] Whitfield, et al., Nature 378 (1995) 379-380.

[4] Underhill, et al., Genome Res. 7 (1997) 996-1005.

[5] Hammer, et al., Am. J. Hum. Genet. 56 (1995) 951-962.

[6] Mathias, et al., Hum. Mol. Genet. 3 (1994) 115-123. 
[7] Veitia, et al., Hum. Genet. 99 (1997) 648-652.

[8] Zerjal, et al., Am. J. Hum. Genet. 60 (1997) 1174-1183.

[9] Seielstad, et al., Hum. Mol. Genet. 3 (1994) 2159-2161.

[10] Righetti, Tyler-Smith, personal communication.

[11] Rosser et al., personal communication.

[12] C. Previderè, et al., Prog. Forensic Genet. 8 (2000) 257-259.

[13] M. Brión, et al., Prog. Forensic Genet. 8 (2000) 263-265.

[14] B. Caeiro, et al., Prog. Forensic Genet. 8 (2000) 287-289.

[15] F.R. Santos, et al., Am. J. Hum. Genet. 64 (1999) 619-628.

[16] B. Myhre Dupuy, et al., Forensic Sci. Int. 117 (2001) 163-173. 